\title{
MAP Reconstruction From Spatially Correlated PET Data
}

\author{
Adam Alessio, Student Member, IEEE, Ken Sauer, Member, IEEE, and Charles A. Bouman, Fellow, IEEE
}

\begin{abstract}
High sensitivity 3-D PET data is often rebinned into 2-D data sets in order to reduce the computation time of reconstructions. The need to precorrect the 3-D data for attenuation, accidentals, scatter, and deadtime effects before rebinning along with the rebinning process itself changes the statistics of the data. This paper presents an approach for finding and using the statistics of Fourier rebinned (FORE) data. In particular, utilizing a space domain representation of FORE, we find the approximate covariance matrix. We also derive an improved estimate of the mean of the rebinned data with a 2-D forward projector that more accurately represents the effect of FORE on the original 3-D PET measurements. In order to incorporate dependent information, we model the data conditioned on the image as a low-order Markov field. This model is based on a quadratic approximation of the log-likelihood of dependent 2-D PET data. The dependence relationship is then incorporated into a novel maximum a posteriori (MAP) 2-D reconstruction method. Initial results show that this method offers modest MSE improvements with a reference image over Poisson-based MAP methods. Results also reveal that the use of only the improved mean leads to significant improvements in reconstructions from FORE data.
\end{abstract}

Index Terms-Correlation, covariance, dependent, FORE, Fourier rebinning, fully 3-D PET, MAP estimation.

\section{INTRODUCTION}

$\mathbf{T}$ HE statistical reconstruction of 3-D positron emission tomography (PET) data represents a formidable computational challenge. Consequently, rebinning methods, of which Fourier rebinning (FORE) [1] is the most popular, are often used to reduce the 3-D data to decoupled sets of 2-D data and then traditional 2-D reconstruction techniques are implemented [2]. This poses a problem because these rebinning methods create 2-D data that do not have the same statistics as directly acquired 2-D data. More specifically, most statistical reconstruction techniques (all EM variations) are based on data modeled as conditionally independent Poisson variables [3], [4]. FORE rebinned data are no longer Poisson because: a) the 3-D data must be fully corrected for accidentals, scatter, attenuation, and deadtime affects before applying FORE and $b$ ) the 2-D data become a linear combination of the 3-D data. This paper will address the often

Manuscript received April 1, 2003; revised June 13, 2003. This work was supported by a Grant from the State of Indiana 21st Century Research and Technology Fund.

A. Alessio was with the Department of Electrical Engineering, University of Notre Dame, Notre Dame, IN 46556 USA. He is now with the University of Washington, Seattle, WA 98112 USA (e-mail: aalessio@u.washington.edu).

K. Sauer is with the Department of Electrical Engineering, University of Notre Dame, Notre Dame, IN 46556 USA (e-mail: sauer@nd.edu).

C. A. Bouman is with the School of Electrical Engineering, Purdue University, West Lafayette, IN 47907 USA.

Digital Object Identifier 10.1109/TNS.2003.817943 ignored reason b) and future extensions of this work will include the effect of a).

A few efforts have been made to approximate the statistics of FORE rebinned data [5], [6]. In this paper, we present a new method for more accurately modeling the rebinned 2-D data as correlated variables and for reconstructing with this new model. We present a technique for finding the correlations introduced through the FORE process. Then, we reconstruct with a maximum a posteriori method using a quadratic approximation of the log-likelihood of this dependent data (MAP-C). We also present an approach for approximating the effect of FORE on the mean of the 2-D data. This modified mean was successfully used in iterative reconstructions and led to the most favorable results.

\section{RATIONALE FOR NEW ESTIMATOR}

Even in idealized 3-D PET systems in which the measured data closely resemble independent random variables, after FORE rebinning, the data are no longer independent. Significant improvements can be expected if one has accurate information about the correlation of the data. The following discussion presents a rough justification for incorporating correlation information into the reconstruction.

In essence, we would like to compare estimators which utilize varying amounts of correlation. In an effort to quantify the mean square error (MSE) of these estimators, we define $\mathbf{y}$ as the $M$ dimensional rebinned sinogram, $\mathrm{x}$ as the ideal $N$ dimensional estimated image and $\tilde{\mathbf{x}}$ as the erroneous estimated image. The linear minimum mean square error estimator would be

$$
\tilde{\mathbf{x}}=E(\mathbf{x})+\tilde{K}_{\mathbf{x y}} \tilde{K}_{\mathbf{y}}^{-1}(\mathbf{y}-E(\mathbf{y}))
$$

where $\tilde{K}_{\mathbf{x y}}$ is the erroneous $N \times M$ cross covariance matrix of $\mathbf{x}$ and $\mathbf{y} \cdot \tilde{K}_{\mathbf{y}}$ is the erroneous covariance function of the data in the sense that it does not include all of the correlation information.

The error covariance, $\tilde{\Upsilon}$, of this erroneous estimator is

$$
\begin{aligned}
\tilde{\mathbf{\Upsilon}} & =E\left[(\mathbf{x}-\tilde{\mathbf{x}})(\mathbf{x}-\tilde{\mathbf{x}})^{\prime}\right] \\
& =K_{\mathbf{x}}-2 \tilde{K}_{\mathbf{x y}} \tilde{K}_{\mathbf{y}}^{-1} K_{\mathbf{y x}}+\tilde{K}_{\mathbf{x y}} \tilde{K}_{\mathbf{y}}^{-1} K_{\mathbf{y}} \tilde{K}_{\mathbf{y}}^{-1} \tilde{K}_{\mathbf{y x}}
\end{aligned}
$$

where $K_{\mathbf{x}}$ is the covariance function of $\mathbf{x}$ and $K_{\mathbf{y}}$ is the ideal covariance function, including all of the correlation information. The error covariance of the ideal estimator is

$$
\Upsilon=K_{\mathbf{x}}-K_{\mathrm{xy}} K_{\mathbf{y}}^{-1} K_{\mathbf{y x}} .
$$

We need to quantify how much larger $\tilde{\Upsilon}$ is than $\boldsymbol{\Upsilon}$. Unfortunately, $K_{\mathbf{y}}^{-1}$ would be difficult to calculate because this is no longer a diagonal matrix representing variances of independent data. Therefore, we resort to a spectral approach. Let $K_{\mathbf{y}}=$ $F \Lambda_{\mathbf{y}} F^{\prime}$ be the K-L decomposition of $K_{\mathbf{y}}$. Under an assumption 
TABLE I

COMPARISON OF APPROXIMATE MSE VALUES FOR DIFFERENT ESTIMATORS

\begin{tabular}{|c|l|c|}
\hline $\begin{array}{c}\text { Number of entries } \\
\text { of } K_{y y} \text { in } \tilde{K}_{y y}\end{array}$ & Description of $\tilde{K}_{y y}$ & $\frac{M \tilde{S} E_{j}}{M S E_{j}}$ \\
\hline \hline 400 & $\begin{array}{l}\text { When estimating } y_{i}, \text { includes } K_{y_{a}} y_{a} \\
\text { where } a=(i-200) \rightarrow(i+200)\end{array}$ & 1.10 \\
\hline 24 & $\begin{array}{l}\text { Includes correlation of } \\
24 \text { closest neighbors of } y_{i}\end{array}$ & 1.30 \\
\hline 8 & $\begin{array}{l}\text { Includes correlation of } \\
8 \text { closest neighbors of } y_{i}\end{array}$ & 1.65 \\
\hline 1 & No correlation included & 3.00 \\
\hline
\end{tabular}

of wide sense stationarity for the Poisson noise in $\mathbf{y}$ (adequate to get a rough idea of the value of the estimator), $F$ is well approximated by the discrete Fourier transform (DFT) matrix and $\Lambda_{\mathrm{y}}$ is a diagonal matrix containing the variances of the DFT coefficients.

Since $\mathbf{y}=\mathbf{P x}+\mathbf{n}$ where $\mathbf{n}$ is the approximately zero mean noise in the Poisson variates and $\mathbf{P}$ is the system matrix, $K_{\mathbf{x y}}=$ $E\left[\mathbf{X}(\mathbf{P X}+\mathbf{N})^{\prime}\right]=E\left[\mathbf{X X}^{\prime}\right] \mathbf{P}^{\prime}+E\left[\mathbf{X N}^{\prime}\right]$. Assuming that the noise and the image are uncorrelated, $K_{\mathbf{x y}}=E\left[\mathbf{X X}^{\prime}\right] \mathbf{P}^{\prime}$. It also should be noted that $K_{\mathbf{x}}=\tilde{K}_{\mathbf{x}}$. When we evaluate (1) at a single pixel $\mathrm{j}$

$$
M \tilde{S} E_{j} \approx k_{x_{j} x_{j}}-2 k_{\mathbf{x} x_{j}}^{\prime} \mathbf{P}^{\prime} F^{*} \tilde{\Lambda}_{\mathbf{y}}^{-1}\left[2 I-\Lambda_{\mathbf{y}} \tilde{\Lambda}_{\mathbf{y}}^{-1}\right] F^{-1} \mathbf{P} k_{\mathbf{x} x_{j}} .
$$

In a similar fashion, the mean square error of the ideal estimator is

$$
M S E_{j} \approx k_{x_{j} x_{j}}-k_{\mathbf{x} x_{j}}^{\prime} \mathbf{P}^{\prime}\left(F^{*} \Lambda_{\mathbf{y}}^{-1} F^{-1}\right) \mathbf{P} k_{\mathbf{x} x_{j}} .
$$

This approach simplifies the mean square error calculation to operations with diagonal matrices. The values of equations (3) and (4) were compared using a 2-D covariance matrix computed from a 70 projection $\times 90$ angle sinogram using the method discussed in the next section. A simple exponential covariance relationship for the image is used in this calculation, providing an adequate representation for a low frequency content image. Table I summarizes the MSE results when differing amounts of correlation are included in $\tilde{K}_{y}$. The last row of the table represents an estimator assuming uncorrelated data which is the current convention in the field. Consequently, with the incorporation of the correlation information of only 8 neighbors, one can expect the MSE to be approximately 55\% that of the uncorrelated estimator. Once again, this assumes the the correct correlation information is known.

\section{Sinogram Covariance AFTER FORE}

FORE essentially converts 3-D sinogram data to 2-D data and consists of a sum of linear operations in frequency space. This process introduces a significant amount of correlation in the data. To our knowledge, no current methods attempt to address this issue.

Using the notation of [1] and [6], with $y_{3 D}()$ representing a fully 3 -D set of sinograms and $y_{2 D}()$ representing a set of re- binned 2-D sinograms, FORE can be written in the space domain as

$$
\begin{gathered}
y_{2 D}(s, \phi, z)=\sum_{s^{\prime} \phi^{\prime}} \sum_{z^{\prime} \Delta^{\prime}} a\left(s-s^{\prime}, \phi-\phi^{\prime}, z, z^{\prime}, \Delta^{\prime}\right) \\
\times y_{3 D}\left(s^{\prime}, \phi^{\prime}, z^{\prime}, \Delta^{\prime}\right)
\end{gathered}
$$

$a()$ is the FORE kernel which describes the contribution of a particular oblique plane, $\Delta^{\prime}$, of plane $z^{\prime}$, to the rebinned plane $z$. $a()$ can be calculated by applying FORE to 3-D impulse sinograms. For instance, if $a(s, \phi, 20,12,3)$ (effect of the third oblique plane of plane 12 on the 2-D plane 20) is needed, set all oblique planes to zero and place a one at $\tilde{y}_{3} D(0,0,12,3)$. Then, apply FORE to $\tilde{y}_{3 D}$ and store the resulting 2-D sinograms in $a(*, *, *, 12,3)$.

Comtat et al. have discussed the FORE kernel [6], but avoided using it due to computational costs. We have simplified calculation and storage issues utilizing the fact that the majority of the energy in the kernel resides near its center. We store and use only the central portion of the kernel.

FORE (5) can be written in matrix notation as

$$
\mathbf{y}_{2 D}=\mathbf{A y}_{3 D}
$$

where $\mathbf{A}$ is the matrix form of convolving by $a()$. Consequently, the covariance of the data has the form

$$
K_{y_{2 D}}=\mathbf{A} K_{y_{3 D}} \mathbf{A}^{\prime}
$$

$K_{y_{3 D}}$ is the covariance matrix of the 3-D data. Since we assume that the 3-D data are conditionally independent, this is a diagonal matrix containing only the estimated variance of the 3-D data. After this transformation, the data are not independent and $K_{y_{2 D}}$ is not diagonal. In fact, using a 279 projection $\times 360$ angle sinogram from laboratory PET data, approximately $39 \%$ of the energy is not on the diagonal. The challenge now resides in efficiently exploiting this covariance information.

Fig. 1 presents the covariance of a data entry, $y_{i}$, with its immediate neighbors. The central point in the figures is the variance of $y_{i}$. After the examination of a variety of scans and FORE setups, we have found that the covariance information has this same basic structure and relationship with neighbors. A cursory inspection reveals that the data are positively correlated with entries at different angles and negatively correlated with entries at different radial distances.

Even with a reduced kernel size, the computation of (7) is significant. This computation requires basically two 2-D convolutions (a pre-and post-convolution). Now that we have a better understanding of the structure of the true $K_{y_{2 D}}$, we believe that faster methods could be used to estimate the 2-D dependent covariance matrix from the $3-\mathrm{D}$ covariance matrix.

\section{Sinogram Mean After FORE}

Along with changes to the covariance, FORE also affects the means of the 2-D data. Currently, reconstruction methods which work with FORE rebinned data use the idealized 2-D projection matrix times the current image to represent the mean, $\tilde{\boldsymbol{\mu}}_{2 D}$, of the rebinned data

$$
\tilde{\boldsymbol{\mu}}_{2 D}=\mathbf{P}_{2 D} \mathbf{x}
$$




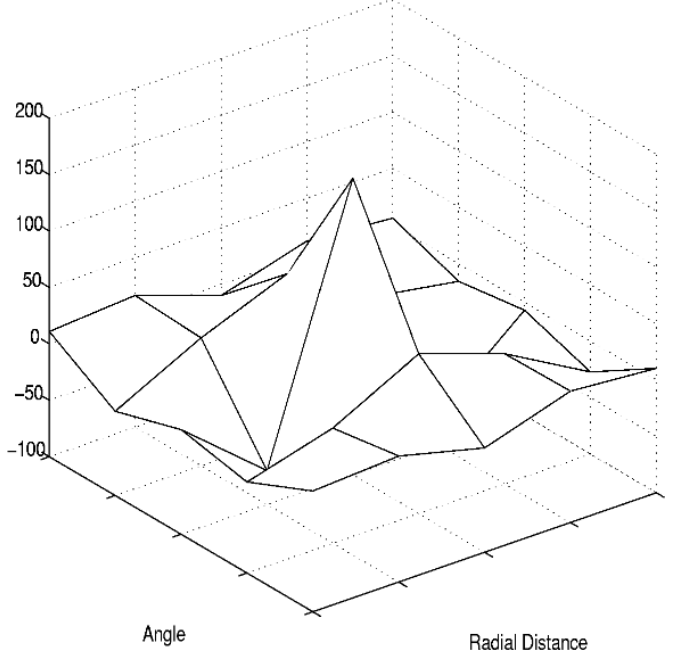

(a)

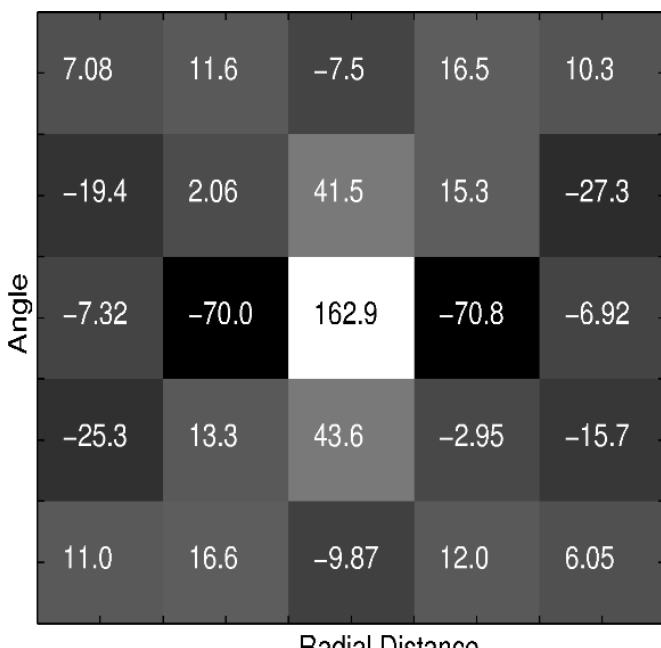

(b)

Fig. 1. Covariance of one data point in center of sinogram with its 24 neighbors.

In actuality, the mean of the FORE rebinned data is better represented as

$$
\boldsymbol{\mu}_{2 D}=\mathbf{A P}_{3 D} \mathbf{x}
$$

This representation acknowledges the fact that FORE has a relatively complex effect on the conditional means of the rebinned projection data. $\mathbf{P}_{3 D}$ is the 3-D forward projection operation consisting of projection information for all oblique planes through the volume image, $\mathbf{x}$. It should be stressed that $\mathbf{P}_{2 D} \neq \mathbf{A P}_{3 D}$.

In traditional iterative reconstructions, each iteration requires the equivalent of one forward and one back projection. The computation of (9) requires the knowledge of the 3-D projection matrix, which is a large part of the reason for avoiding fully 3-D reconstruction and adopting FORE. Therefore, a simplification of the (9) needs to be adopted.

It is not possible to form an exact expression representing how FORE influences the idealized 2-D projection matrix. FORE rebinned data are found from the summative influence of all 3-D planes convolved with their corresponding FORE kernel.
We adopt an approach which approximates the 3-D influence with a new kernel containing the summation of all the FORE kernels for a given direct plane, $z$. In this approach, the means undergo a similar process as the data; the drawback is that we do not have access to the variation in contribution from different 3-D planes. Positive results further support this approach.

Specifically, we calculated a new convolution kernel that includes the summative effects of all of the planes of the FORE kernel

$$
\hat{a}(s, \phi, z)=\sum_{z^{\prime} \Delta^{\prime}} a\left(s, \phi, z, z^{\prime}, \Delta^{\prime}\right) .
$$

Then, we convolved this kernel with the idealized projection matrix

$$
\hat{\mathbf{P}}_{2 D}(s, \phi, z)=\sum_{s^{\prime} \phi^{\prime}} \hat{a}\left(s-s^{\prime}, \phi-\phi^{\prime}, z\right) \mathbf{P}_{2 D}\left(s^{\prime}, \phi^{\prime}, z\right) .
$$

The improved projection matrix, $\hat{\mathbf{P}}_{2 D}$, can be computed once for a given system geometry and then stored. Even computation on the fly is reasonable considering, as we mentioned before, that the kernels can be reduced in size because the majority of its energy resides in the central portion. Equipped with this modified projection matrix, the approximate mean of the FORE rebinned data is

$$
\hat{\boldsymbol{\mu}}_{2 D}=\hat{\mathbf{P}}_{2 D} \mathbf{x}
$$

Another way to view this new mean is as a blurred version of the ideal mean. FORE has a point spread function that blurs the data in a specified manner. This new mean incorporates this blur into the system model.

\section{RECONSTRUCTING WITH COVARIANCE INFORMATION}

The approximate covariance matrix would make the ideal weighting matrix in reconstruction methods such as penalized, weighted least-square (PWLS). Unfortunately, this nondiagonal matrix would need to be inverted, which is not feasible for reasonably sized data sets.

In our formulation, we assume that the 2-D rebinned data, conditioned on the image, is a Markov random field. Since the exact form of the likelihood function after FORE appears intractable, we use the first and second moments to form the second order Taylor series approximation of the log-likelihood.

Initially, the 3-D likelihood function is Poisson and has a Taylor series approximation [7]

$$
\begin{aligned}
\log \mathcal{P}\left(\mathbf{Y}_{3 D}=\mathbf{y}_{3 D} \mid \mathbf{x}\right) \approx c\left(\mathbf{y}_{3 D}\right) \\
\quad-\frac{1}{2}\left(\mathbf{y}_{3 D}-\mathbf{P}_{3 D} \mathbf{x}\right)^{\prime} \Lambda^{-1}\left(\mathbf{y}_{3 D}-\mathbf{P}_{3 D} \mathbf{x}\right)
\end{aligned}
$$

where $\Lambda=\operatorname{diag}\left\{y_{i}^{-1}\right\}$ and is the 3-D weighting matrix. It should be noted that although (13) appears to be a Gaussian likelihood, it is the second term of the Taylor series expansion of the Poisson likelihood. Please note that all of the $K \mathrm{~s}$ and functions of $K$ that follow are functions of $y$ and therefore not true covariance matrices. But, all of the following algebra holds as if the Gaussian view (which uses covariance matrices) were used. 
When the data are transformed to 2-D through the linear operation (6), it assumes the form

$$
\begin{aligned}
\log \mathcal{P}\left(\mathbf{Y}_{2 D}=\mathbf{A y}_{3 D} \mid \mathbf{x}\right) \approx c\left(\mathbf{y}_{2 D}\right) \\
\quad-\frac{1}{2}\left(\mathbf{y}_{2 D}-\mu_{2 D}\right)^{\prime} K_{y_{2 D}}^{-1}\left(\mathbf{y}_{2 D}-\mu_{2 D}\right) .
\end{aligned}
$$

$K_{y_{2 D}}$ is an approximation of the 2-D covariance matrix or, more accurately, the transformation of the 3-D weighting matrix. As stated above, the inverse of $K_{y_{2 D}}$ is not feasible, and (14) can be further simplified using a Markov assumption. That is, for a given 2-D sinogram entry $y_{i}$ and its neighbors $\mathbf{n}_{i}=$ $\left\{n_{1} \ldots n_{B}\right\}$

\begin{tabular}{|c|c|c|}
\hline$n_{1}$ & $n_{2}$ & $n_{3}$ \\
\hline$n_{4}$ & $y_{i}$ & $n_{5}$ \\
\hline$n_{6}$ & $n_{7}$ & $n_{8}$ \\
\hline
\end{tabular}

we need to find the distribution

$$
f\left(y_{i} \mid \mathbf{x}, \mathbf{n}_{i}\right)=\frac{f\left(y_{i}, n_{1}, \ldots, n_{B} \mid \mathbf{x}\right)}{f\left(n_{1}, \ldots, n_{B} \mid \mathbf{x}\right)} .
$$

In the following, $K_{f g}$ is the weighting for sinogram entry $f$ and $g, \mathbf{K}_{\mathbf{N}_{\mathbf{i}}}$ is the approximate $B \times B$ weighting matrix of the neighbors

$$
\begin{aligned}
\mathbf{K}_{\mathbf{N}_{\mathbf{i}}} & =\left[\begin{array}{ccccc}
\sigma_{n_{1}}^{2} & K_{n_{1} n_{2}} & & \ldots & \\
K_{n_{1} n_{2}} & \sigma_{n_{2}} & K_{n_{2} n_{3}} & & \\
& \vdots & \ddots & & \\
& & & & \sigma_{n_{B}}^{2}
\end{array}\right] \\
\mathbf{K}_{\mathbf{y}_{\mathbf{i}} \mathbf{N}} & =\left[K_{y_{i} n_{1}} K_{y_{i} n_{2}} \ldots K_{y_{i} n_{B}}\right]
\end{aligned}
$$

$\mathbf{K}_{\mathbf{N y}_{i}}=\mathbf{K}_{\mathbf{y}_{i} \mathbf{N}^{\prime}}$, and $Q_{i}=\sigma_{y_{i}}^{2}-\mathbf{K}_{\mathbf{y}_{\mathbf{i}} \mathbf{N}} \mathbf{K}_{\mathbf{N}_{\mathbf{i}}}^{-1} \mathbf{K}_{\mathbf{N y}_{\mathbf{i}}}$. We know $f\left(n_{1}, \ldots, n_{B} \mid \mathbf{x}\right) \approx c_{1}(y) \exp \left(-\frac{1}{2}\left(\mathbf{n}_{\mathbf{i}}-\boldsymbol{\mu}_{\mathbf{n}_{\mathbf{i}}}\right)^{\prime} \mathbf{K}_{\mathbf{N}_{\mathbf{i}}}^{-1}\left(\mathbf{n}-\boldsymbol{\mu}_{\mathbf{n}_{\mathbf{i}}}\right)\right)$

and

$$
\begin{aligned}
& f\left(y_{i}, n_{1}, \ldots, n_{B} \mid \mathbf{x}\right) \\
& \quad=f(\mathbf{v} \mid \mathbf{x}) \\
& \quad \approx c_{2}(y) \exp \left(-\frac{1}{2}\left(\mathbf{v}-\mu_{\mathbf{v}}\right)^{\prime} \mathbf{K}_{\mathbf{V}}^{-1}\left(\mathbf{v}-\mu_{\mathbf{v}}\right)\right) .
\end{aligned}
$$

Subtracting the exponential terms and simplifying yields

$$
\begin{aligned}
& f\left(y_{i} \mid \mathbf{x}, \mathbf{n}_{i}\right) \approx c_{3}(y) \\
& \quad \times \exp \left(-\frac{1}{2} \frac{\left[\left(y_{i}-\mu_{i}\right)-\mathbf{K}_{\mathbf{y}_{\mathbf{i}} \mathbf{N}} \mathbf{K}_{\mathbf{N}_{\mathbf{i}}}^{-1}\left(\mathbf{n}_{\mathbf{i}}-\boldsymbol{\mu}_{\mathbf{n}_{\mathbf{i}}}\right)\right]^{2}}{\mathbf{Q}_{\mathbf{i}}}\right) .
\end{aligned}
$$

Under the conditional Markov assumption, the approximation of the likelihood function simplifies to the form

$$
\tilde{f}(\mathbf{y} \mid \mathbf{x}) \propto \prod_{i=1}^{M} \exp \left(-\frac{1}{2} \frac{\left[\left(y_{i}-P_{i *} \mathbf{x}\right)-\mathbf{Z}_{i}\left(\mathbf{n}_{\mathbf{i}}-\mathbf{P}_{\mathbf{n}_{\mathbf{i}} *} \mathbf{x}\right)\right]^{2}}{Q_{i}}\right) .
$$

Here, $\mathbf{Z}_{i}=\mathbf{K}_{\mathbf{y}_{\mathbf{i}} \mathbf{N}} \mathbf{K}_{\mathbf{N}_{\mathbf{i}}}^{-1}$ is a $1 \times B$ vector, $P_{i *} \mathbf{X}=\sum_{k=1}^{N} P_{i k} x_{k}$ represents the contribution of all emission sites to projection $i$, and $\mathbf{P}_{\mathbf{n}_{i} *} \mathbf{x}$ is a $B \times 1$ vector containing the contribution of emission sites to the neighbors of projection $i$. We apply this approach with both the idealized $\mathbf{P}_{2 D}$ and the improved $\hat{\mathbf{P}}_{2 D}$
TABLE II

COMPUTATIONAL COMPARISON OF 2-D RECONSTRUCTION METHODS, LISTING MEASURES OF COMPUTATIONAL TIME PER FUll ITERATION. $M_{0}$ IS THE Number of NONZERo ProjeCtions ASSOCIATED With EACH OF THE $N$ PiXels. For THE TIMe STUdy, MAP-C Used $B=8$

\begin{tabular}{|r||c|c|c|}
\hline Algorithm & \# of multi. \& div. & \# of matrix reads & time (sec.) \\
\hline FBP & $M_{0} N$ & 1 & NA Full Recon:33.0 \\
\hline EM & $2 M_{0} N$ & 2 & 29.3 \\
\hline MAP-ICD & $4 M_{0} N$ & 2 & 30.3 \\
\hline MAP-D & $(2 B+4) M_{0} N$ & 2 & 83.7 \\
\hline
\end{tabular}

projection matrices. $Q_{i}$ is a function of $y$ which preserves the key statistical properties inherited from the Poisson likelihood of the original 3-D data.

We have chosen to use a regularized estimation method in order to improve low signal-to-noise ratio cases. For all of the methods reviewed here we will use the generalized Gaussian MRF (GGMRF) model [8], denoted as $G(x)$, as the regularization term. When this is combined with the log of (18), the overall objective function is

$\Phi(\mathbf{x})=G(x)+\sum_{i=1}^{M}\left[\frac{1}{2} \frac{\left[\left(y_{i}-P_{i *} \mathbf{x}\right)-\mathbf{Z}_{i}\left(\mathbf{n}_{\mathbf{i}}-\mathbf{P}_{\mathbf{n}_{i *}} \mathbf{x}\right)\right]^{2}}{Q_{i}}\right]$.

The maximization of this objective function with the ICD/Newton-Raphson method found in [7] will be termed "MAP-Correlated" or MAP-C.

\section{IMPLEMENTATION AND RESULTS}

The discussion in this paper leads to three new approaches for reconstructing FORE rebinned data. From simplest to more complex, one method would be to use traditional statistical methods with an improved calculation of the mean (12). This essentially requires a modified projection matrix which can be easily calculated at the beginning of the reconstruction and leads to a minor increase in overall reconstruction time.

Secondly, the MAP-C method consists of the following steps:

1) generate FORE kernels for a particular 3-D data size;

2) collect data in fully 3-D mode;

3) if desired, correct 3-D data for attenuation, deadtime, detector efficiencies, and decay;

4) estimate 3-D weighting matrix and transform into 2-D with FORE kernels (7);

5) FORE rebin data [1];

6) minimize dependent objective function(19).

Along with improving the quality of the reconstruction from rebinned data, another primary goal of this estimator is to provide a faster alternative to fully 3 -D reconstruction. Table II compares the computational load of the proposed MAP-C algorithm with other two dimensional reconstruction algorithms. The time column presents the average time for one iteration of a $128 \times 128$ image using a $279 \times 360$ data set on a Sun Blade 1000 Workstation with $512 \mathrm{Mb}$ of RAM. $P$ is a sparse matrix containing $M_{0} N$ nonzero entries. MAP-C requires a $2 B M_{0} N$ more multiplies than traditional MAP-ICD due to the matrix multiplications necessary in the optimization. This may seem exceptionally slow, but the multiplications do not dominate the computation time during each iteration as shown in the similar times for 


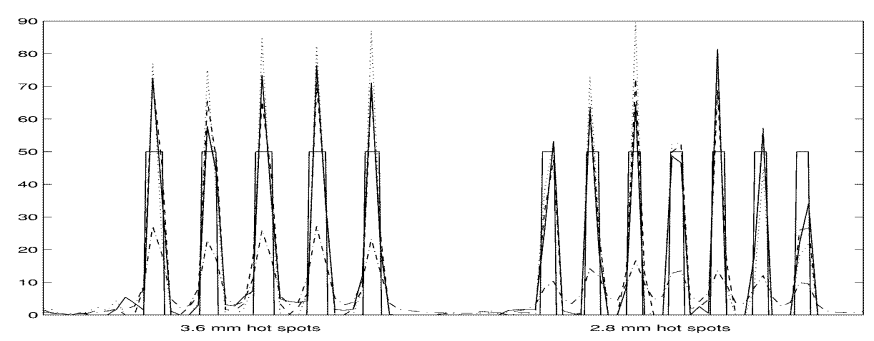

Fig. 2. Profile of horizontal line 73 from simulated phantom. Reference image (solid); FBP (dashdot); Poisson MAP (dotted); MAP-with improved mean (dashed); MAP-C-with improved mean (solid)

EM and MAP-ICD and in the fact that MAP-C has $5 \times$ the multiplications as MAP-ICD, but only requires $3 \times$ the time.

Even though the computation per iteration is reasonable, the setup time for MAP-C is significant and remains an area for improvement. MAP-C requires two significant steps before beginning to optimize the objective. First of all, the transformation of the 3-D weighting to 2-D is currently a nonoptimal $O\left(M^{2} K_{s}^{2} Z_{p l}^{2}\right)$ process where $M$ is the size of the sinogram, $K_{s}$ is the size of one dimension of the FORE kernel, and $Z_{p l}$ is the number of detector planes. Secondly, MAP-C must also compute $Z_{i}$ for all $M$ projections which involves $M B \times B$ matrix inversions (for a total complexity of $O\left(M * B^{3}\right)$ ). Even with these steps, MAP-C remains substantially faster than fully 3-D algorithms.

The final method would be a combination of MAP-C with the improved mean, representing the most tailored approach for working with the statistics of FORE rebinned data. The addition of the improved mean does not significantly affect the computation time so the previous discussion holds.

Testing has revealed that the MAP-C method is visually comparable to the traditional Poisson model MAP. It also offers slight improvements in the MSE error between the true image and the reconstructions. For all trials, we included only eight neighbors of $y_{i}(B=8)$. Initially, we compared reconstructions of simulated 3-D Shepp phantoms. These were forward projected into $180 \times 150$ sinograms and Poisson noise and uniform scatter were introduced. With the same prior influence, the MAP-C reconstruction had $80 \%$ of the MSE of the original MAP reconstruction.

Fig. 3 shows reconstructions from a simulated 3-D Derenzo-like phantom. The geometry for this simulation resembled a small animal scanner, featuring 24 rings of detectors with a $38 \mathrm{~cm}$ diameter and a $23 \mathrm{~cm}$ transaxial FOV. The $15 \mathrm{~cm}$ axial FOV resulted in an axial aperture of $22^{\circ}$. The phantom spanned seven planes and was 3-D forward projected into $279 \times 360$ sinograms. Poisson noise and $20 \%$ uniform scatter were added. No other physical effects (attenuation, deadtime, etc.) were incorporated. For the MAP-C methods, the approximate 2-D covariance matrix was computed from the 3-D data through the use of $30 \times 30$ FORE kernels. For all reconstructions, the sinograms were FORE rebinned into 47 2-D planes. The filtered backprojection reconstruction used an $80 \%$ cutoff Hanning filter. All of the MAP methods were run to convergence.

In all of the reconstructions, there is a strong angular blurring at larger radial distances. The FORE approximation becomes

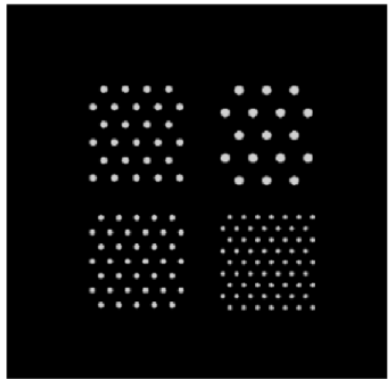

(a)

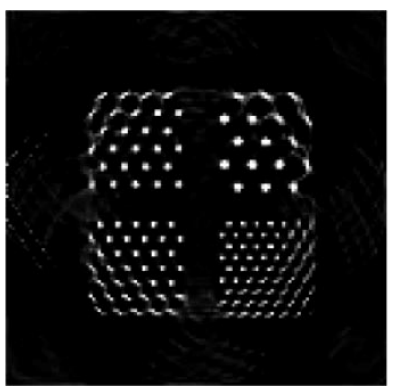

(c)

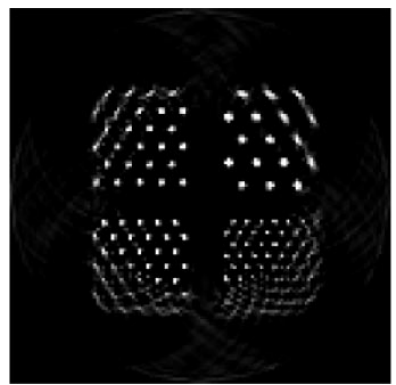

(e)

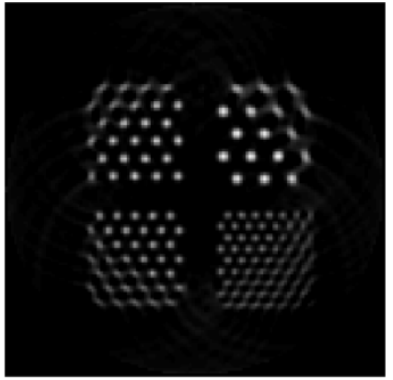

(b)

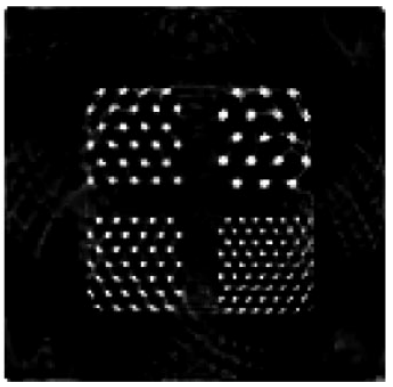

(d)

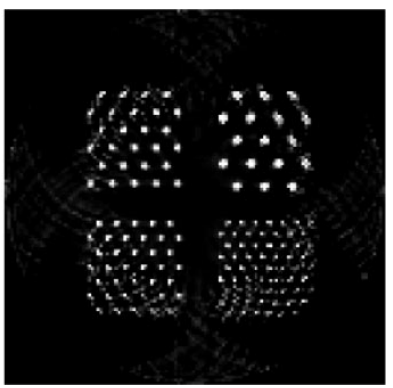

(f)
Fig. 3. $128 \times 128$ Reconstructions of Simulated Derenzo-like phantom from FORE rebinned $279 \times 360$ data. Consists of four quadrants with $5.6 \mathrm{~mm}$ diameter hot spots to $2.8 \mathrm{~mm}$ hot spots. (a) Phantom. (b) FBP. (c) MAP. (d) MAP-improved mean. (e) MAP-C. (f) MAP-C improved mean.

less accurate as the axial aperture increases. Our choice of a large axial aperture is some of the cause for this degradation at the edge of the FOV. Also, in short, in order to validate the FORE approximation, this rebinning algorithm clips angular frequency information in a region defined by the FOV due to the Radon transform consistency condition [1]. This clipping causes a loss of some valuable high angular frequencies near the edge of the FOV resulting in some axial blurring. This data set, which purposefully highlights the faults of FORE, offers an opportunity to see improvements upon its conventional application.

Once again, the MAP-C method is visually similar to the traditional Poisson model MAP. The addition of the improved mean yielded the most significant improvement. Specifically, the "MAP-Improved Mean" reconstruction almost completely removed the angular blurring occurring around the edge of the FOV.

Fig. 2 reveals the profile from a central portion of these reconstructions along a vertical line. All of the MAP methods are equally capable of resolving the lower right, $2.8 \mathrm{~mm}$ hot spots (FBP is not). The "MAP-C-improved mean" and "MAP-improved mean" are somewhat more consistent at reaching the true 


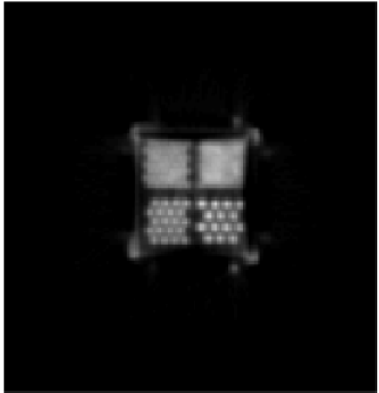

(a)

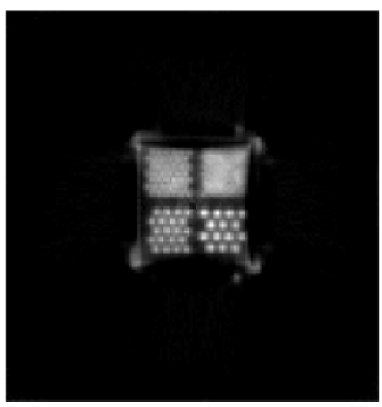

(c)

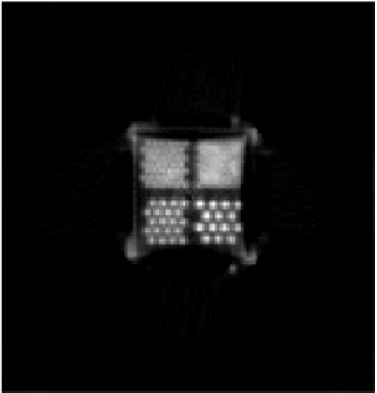

(b)

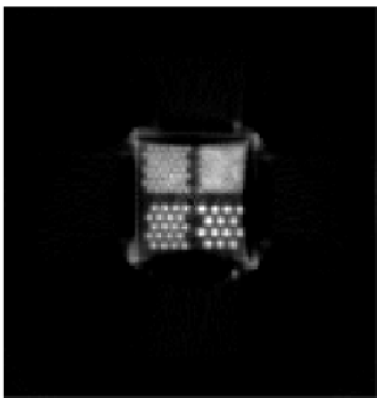

(d)

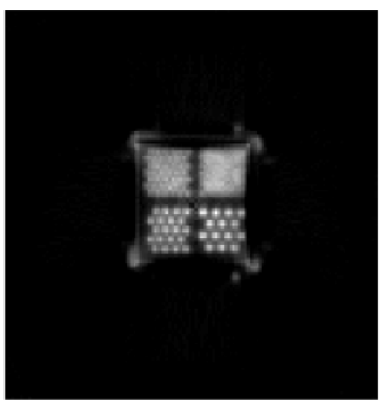

(e)

Fig. 4. $128 \times 128$ Reconstructions of Real Derenzo-like phantom from FORE rebinned $279 \times 360$ data. Consists of 4 quadrants with $4.0 \mathrm{~mm}$ diameter hot spots to $1.6 \mathrm{~mm}$ hot spots. (a) FBP. (b) Poisson MAP. (c) MAP-improved mean. (d) MAP-C. (e) MAP-C improved mean.

value of the hot spots particularly at the right edge where MAP blurs the edge of the FOV.

For this simulated phantom, MAP-C yields reconstructions with $90 \%$ the MSE of MAP. This value, along with the $80 \%$ from the Shepp trials, are not as positive as the $55 \%$ expected from table tab:MSE. Given that this expected figure is based on a stationarity assumption for the data, knowledge of the true covariance information, and a simple low frequency model for the image some discrepancy is to be expected. Interestingly, the "MAP-improved mean" produces reconstructions with superior visual quality and with a MSE of $81 \%$ of the standard MAP.

These methods were also tested with clinical data from the IndyPET-II scanner [9] on a phantom similar to the simulated version. $6.9 \times 10^{8}$ events were collected in fully 3 -D mode and stored in 216 oblique $279 \times 360$ sinograms. The data were precorrected for attenuation, deadtime, and detector efficiency prior to being FORE rebinned into 47 direct sinograms. The 2-D attenuation correction factors were applied to the rebinned data and incorporated into the system matrix as described for the attenuation weighting methods in [5]. While reconstructions can be improved by including scatter and accidentals, we decided to maintain simplicity in order to see what improvements can be made with just an exploration of the correlations in the data. Fig. 4 displays reconstructions of this data.

Once again, the "MAP-improved mean" provides the best visual quality. One can begin to resolve the $2.4 \mathrm{~mm}$ hot spots in the second quadrant. MAP-C provides a slightly sharper contrast than the traditional MAP, but in essence these reconstructions visually comparable.

\section{CONCLUSION}

Theoretically, the introduction of correlation information should enhance reconstructions. Modest gains in terms of MSE with a reference image have been presented. Results show that the minor adjustment to the 2-D mean (12) can lead to significant improvements in reconstructions. These improvements are especially noticeable when the object approaches the edge of the FOV.

Current visual results do not strongly support the use of MAP-C. Several stages of this approach need improvement. For instance, we need to improve the choice of 3-D weighting matrices especially with corrected 3-D data. Moreover, we hope to speed and refine the transformation of the 3-D weighting matrix to the 2-D domain. Most importantly, further testing may reveal that the inclusion of more neighbors is necessary in order to yield higher quality reconstructions. The introduction of more neighbors will increase reconstruction time leading to the need for faster optimization methods.

Another avenue for improvement involves including the correlation introduced across multiple planes as opposed to just within a 2-D sinogram. FORE consists of a linear interpolation between rebinned slices which clearly adds correlation. We have shown that significant improvements are possible when this axial information is incorporated into the mean of the statistical model [10].

At this point, we recommend modifying the 2-D projection matrix in all statistical methods which work with FORE rebinned data. Further study may lead to a method which will more effectively incorporate the 2-D dependent covariance information.

\section{ACKNOWLEDGMENT}

The authors would like to thank N. Rouze, M. Miller, and G. Hutchins, Indiana University, Bloomington, IN, for the use of data from the IndyPET-II scanner.

\section{REFERENCES}

[1] M. Defrise, P. Kinahan, D. Townsend, C. Michel, M. Sibomana, and D. Newport, "Exact and approximate rebinning algorithms for 3-D PET data," IEEE Trans. Med. Imaging, vol. 16, pp. 145-158, Apr. 1997.

[2] M. Krzywinski, V. Sossi, and T. Ruth, "Comparison of FORE, OSEM, and SAGE algorithms to 3-DRP in 3-D PET using phantom and human subject data," IEEE Trans. Nucl. Sci., vol. 46, pp. 1114-1120, Aug. 1999.

[3] H. Hudson and R. Larkin, "Accelerated image reconstruction using ordered subsets of projection data," IEEE Trans. Med. Imaging, vol. 13, pp. 601-609, Dec. 1994.

[4] J. Fessler and A. Hero, "Penalized maximum-likelihood image reconstruction using space-alternating generalized EM algorithms," IEEE Trans. Image Processing, vol. 4, pp. 1417-1429, Oct. 1995. 
[5] X. Liu, C. Comtat, C. Michel, P. Kinahan, M. Defrise, and D. Townsend, "Comparison of 3-D reconstruction with 3-D-OSEM and with FORE+OSEM for PET," IEEE Trans. Med. Imaging, vol. 20, pp. 804-814, Aug. 2001.

[6] C. Comtat, P. Kinahan, M. Defrise, C. Michel, and D. Townsend, "Fast reconstruction of 3-D PET data with accurate statistical modeling," IEEE Trans. Nucl. Sci., vol. 45, pp. 1083-1089, June 1998.

[7] C. A. Bouman and K. Sauer, "A unified approach to statistical tomography using coordinate descent optimization," IEEE Trans. Image Processing, vol. 5, pp. 480-492, Mar. 1996.
[8] — "A generalized Gaussian image model for edge-preserving map estimation," IEEE Trans. Image Processing, vol. 2, pp. 296-310, July 1993.

[9] N. Rouze, K. Stantz, and G. Hutchins, "Design of IndyPET-II, high-resolution, high-sensitivity research scanner," in Proc. IEEE Nuclear Science Symp./Medical Imaging Conf., San Diego, CA, 2001.

[10] A. Alessio, K. Sauer, and C. Bouman, "PET statistical reconstruction with modeling of axial effects of FORE," in Proc. 2003 IEEE Nuclear Science Symp./Medical Imaging Conf., to be published. 